\title{
Open Framework for Combined Pedestrian Detection
}

\author{
Floris De Smedt and Toon Goedemé \\ EAVISE, KU Leuven, Sint-Katelijne-Waver, Belgium \\ firstname.lastname@esat.kuleuven.be
}

Keywords: $\quad$ Pedestrian Detection, Real-time, Framework.

\begin{abstract}
Pedestrian detection is a topic in computer vision of great interest for many applications. Due to that, a large amount of pedestrian detection techniques are presented in current literature, each one improving previous techniques. The improvement in accuracy in recent pedestrian detection, is commonly in combination with a higher computational requirement. Although, recently a technique was proposed to combine multiple detection algorithms to improve accuracy instead. Since the evaluation speed of this combination is dependent on the detection algorithm it uses, we provide an open framework that includes multiple pedestrian detection algorithms, and the technique to combine them. We show that our open implementation is superior on speed, accuracy and peak memory-use when compared to other publicly available implementations.
\end{abstract}

\section{INTRODUCTION}

Pedestrian detection is a subject of great interest in recent literature. Over the years a lot of work has been performed on both speed (Dollár et al., 2010; Benenson et al., 2012; Dollár et al., 2014; De Smedt et al., 2013), and accuracy (Benenson et al., 2013; Park et al., 2010). Although most of these techniques are based on similar detection techniques, the contribution they propose are applied on a single pedestrian detector. These improvements come mostly with an extra computational requirement which is not always available. Recently, (De Smedt et al., 2014) proposed an alternative technique to improve accuracy by combining the detection results of multiple detectors. Their work uses only the detection results neglecting the evaluation speed of the detection algorithms themself. In this paper we propose an open framework that provides the whole pipeline, from the image to running multiple object detection algorithms and finally the combination of their results. By reducing the computational requirement of the pedestrian detection algorithms, the combination they are part of will also come at a limited computational cost. Therefor we compare our algorithms with other publicly available implementations on speed, accuracy and peak memory-use, and show that our implementations turn out to be superior based on these criteria.

The paper is structured as follows: In section 2 we give an overview of existing literature. In section 3 we discuss the implementation of the pedestrian de- tection algorithms we implemented. In section 4 we give a detailed insight on how to combine the detection results. And finally we conclude in section 5 .

\section{RELATED WORK}

Due to the wide applicability of pedestrian detection in a variety of applications (traffic, surveillance, robotics and safety), their has been a lot of research on this topic. In 2005 (Dalal and Triggs, 2005) proposed a technique of using gradient information for this task. They use a grid of HOG-features trained with a linear Support Vector Machine, which imposed impressive detection results on the INRIA pedestrian dataset. Datasets from more realistic conditions (such as the Caltech pedestrian dataset (Dollár et al., 2012b)) showed room for improvement on both accuracy and speed.

We can distinguish two fundamental techniques to improve the accuracy of this detector. The model can be extended, so instead of using a rigid model that searches only for the object as a whole, the model also includes parts (e.g. the limbs of a person). By allowing a limited position deviation of the parts relatively to the root model, a certain pose variation is allowed (Felzenszwalb et al., 2008). On the other hand, one can enrich the features used for pedestrian detection by using color information next to gradient information. This has been done in Integral Channel Features proposed by (Dollár et al., 2009). 
(Dollár et al., 2012b) and (Benenson et al., 2014) give an overview of over 40 different pedestrian detection algorithms in literature, discussing their evaluation methodology and accuracy. Here we see that most of these techniques are based on the two algorithms we discussed before. One of the most accuracte algorithms is described in (Benenson et al., 2013), where each step of the training process of Integral Channel Features is evaluated and optimised. This detector forms the base of (Mathias et al., 2013) which copes with occlusion.

To allow the use of pedestrian detection in applications, both speed and accuracy need to be very high. The speed can be improved by (a combination of) three approaches. One can use more capable hardware such as GPU or multi-core CPU to exploit parallellisation, as has been done by (Benenson et al., 2012; De Smedt et al., 2012; De Smedt et al., 2013). Constraining the appearances to a limitted amount of sizes and positions by using ground plane assumption and/or tracking reduces the searching space (De Smedt et al., 2013; Benenson et al., 2012), and thereby the calculation time needed. A last option is to optimise the algorithms themself. Some examples are approximating features from calculated ones (Dollár et al., 2014; Dollár et al., 2010), learn a model for each scale (Benenson et al., 2012), using a cascaded evaluation (Felzenszwalb et al., 2010a; Bourdev and Brandt, 2005) and using Crosstalk Cascade where the detection results at nearby locations is exploited (and so reducing the amount of locations evaluated) (Dollár et al., 2012a).

To allow the use of these algorithms, some of the implementations are made publicly available. The implementations of (Benenson et al., 2012; Benenson et al., 2013; Benenson et al., 2014) are combined in a single framework ${ }^{1}$. This framework is mostly directed to using a (modern) GPU for fast processing, while using very accurate detection approaches. (Dubout and Fleuret, 2012) made the code available $^{2}$ for an optimised version of the Deformable Part Model detector, as described in (Felzenszwalb et al., 2008). In contrast to (Felzenszwalb et al., 2010a), where a cascade-approach is used for speed improvement, they make use of multi-threading and apply convolution in the Fourier-domain as a dotproduct. Altough, this implementation comes at the cost of high memory-use, as we will point out in section 3. The availability of GPU and high memory restricts the applicability of these frameworks on for example embedded systems. In this paper we propose a complementary framework focussing on cpu-

\footnotetext{
${ }^{1}$ https://bitbucket.org/rodrigob/doppia

${ }^{2}$ http://charles.dubout.ch/en/coding.html
}

implementations at limited memory-use.

Recently (De Smedt et al., 2014) proposed a technique to even further improve the accuracy. Instead of tuning a single object detection algorithms, they combine the strenghts of different algorithms by combining the detection results. They use the measurement of confidence (how trustworthy is a detection of a certain detector) and complementarity (how different are two detectors, so how likely is it they result in the same detections) to combine the detecions scores in a weighted sum. Since the score-range between detectors can vary drastically, they use a normalisation step based on the average and mean of the scores. In this paper we apply this technique on our implementations as an alternative for a computational intensive single tuned algorithm.

\section{IMPLEMENTATION OF OBJECT DETECTION ALGORITHMS}

Each choice that is made in the construction of an object detection algorithm influences the final results it will obtain. In (Benenson et al., 2013) for example, is shown how optimising each step of the training procedure of Integral Channel Detector (Dollár et al., 2009) can lead to a drastic improvement in accuracy (coined the Roerei-detector). The distinguishability of features and structure of the model is bound to the dimension reduction it imposes on the huge searching space to find pedestrians. Next to that, is also the computational complexity, and by consequence the evaluation speed, of great importance when realtime execution is required. As is shown in (De Smedt et al., 2014), each detector has its own strenghts and weaknesses due to the differences in design, and will also lead to different detection results. They provide a technique to combine the detection results of multiple pedestrian detectors to improve detection accuracy, in contrast to the traditional approach of improving a single detector with higher computational cost as a consequence. The cost of combining multiple detection algorithms depends on the algorithms themselves. In this section we describe the pedestrian detection algorithms we implemented in our framework, and compare them on speed, peak memory-use and accuracy. Based on this comparison, we make pairwise combinations of pedestrian detection algorithms in section 4. 


\subsection{Traditional Object Detection Approach}

The approach to distinguish the appearance of an object from the background is a difficult task, mostly solved in the same sequence of steps. The image is rescaled multiple times, to obtain the detections at multiple scales. Rescaling the model (or obtaining a model for different scale appearances of the object) is discussed in (Benenson et al., 2012), and is far more complex than rescaling the image instead. For each scale certain features are calculated to emphasise properties of the image capable to distinguish the object from the background. The last step is to express the similarity between the features and a pretrained model as a numeric value. All the detections with a score above a chosen threshold will be seen as containing the object, where the ones below the threshold are treated as background information. A high threshold will lead to only a few detections, but also less false detections (a high precision), where a low threshold ensures that more appearences of the object will be found, but also more false detections will be made (high recall). The accuracy of an object detection algorithm for different thresholds can be expressed in a precision-recall curve. We use this kind of curve extensively in this paper, an example can be seen in figure 3 .

\subsection{Histogram of Oriented Gradients}

The first algorithm we integrated in our framework is Histogram of Oriented Gradients (HOG). This technique, described in (Dalal and Triggs, 2005) for pedestrian detection, makes use of contrast information of the image to recognise the appearance of pedestrians. The model used here, forms the rootmodel for the Deformable Part Models-detector and is shown in figure 2 at the left. The implementation we use is part of OpenCV. For speed improvement, they use a very similar approach as we do, by evaluating the layers of the scale-space-pyramid in parallel. For integration in our framework, we convert the detection results to our format. To allow a fair comparison with the other algorithms, we use our own NonMaximum suppression implementation instead of the one provided by OpenCV.

\subsection{Integral Channel Features}

As described in section 2, the Integral Channel Features detector makes use of multiple features for object detection. Each feature is presented as a channel. In the original implementation, 10 channels are used (6 gradient orientation, 1 gradient magnitude and 3 colour channels). These are shown in figure 1. The model is constructed from a selection of rectangles containing the sum of the intensity values from a channel. The rectangles are selected from a huge pool of random rectangles using Adaboost. To improve the speed, we use softcascade (Bourdev and Brandt, 2005) so that after the evaluation of each feature, the current score is required to reach a pre-determined treshold to continue the evaluation at that location. To find the annotation, it is required that the detection bounding box has an overlap of at least $50 \%$ with the annotation. The selection of the threshold used for softcascade determines the resulting balance between accuracy and speed. A high threshold will lead to more pruning, so higher speed, where a lower threshold is more indulgent and will allow more detections to reach the final stage at the cost of evaluation speed.

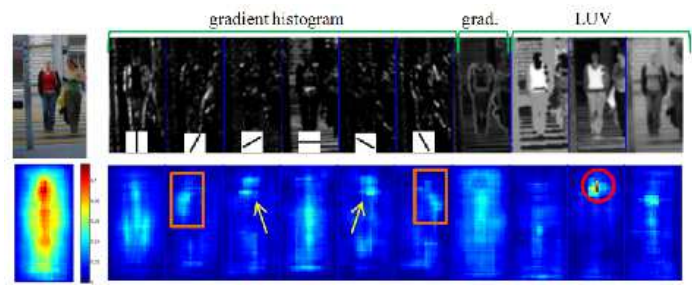

Figure 1: The channels used in the original implementation of (Dollár et al., 2009).

To calculate the features, we use the code released as part of the ACF-implementation of (Dollár et al., 2014), which is heavily optimised for cpu (Dollár, 2013). To improve accuracy, the model used for evaluation is trained with an extra space around the annotation. After detection, the bounding box is altered to the original dimensions, which better fits the object. The accuracy and evaluation speed of our implementation are discussed in subsection 3.5.

\subsection{Deformable Part Models}

The Deformable Part Model detector we use is based on the vanilla implementation used by (De Smedt et al., 2012) and (De Smedt et al., 2013). It is a $\mathrm{C}++$-port of the matlab implementation of DPMv4 released in (Felzenszwalb et al., 2010b). Deformable Part Models can be seen as an extension of the HOGmodel used by (Dalal and Triggs, 2005) with part models. The evaluation of the model can be divided in the search for the root model, representing the object as a whole, and the search for parts (e.g. the limbs of a person). The location of the part models relative to the position of the root model can deviate a litte, to allow a certain pose variation in contrast to rigidmodel detectors. The model we use is trained on the 
INRIA-dataset and is visualized in figure 2. Since the part models are a more detailed element of the object, they are searched for at twice the resolution as the root model. This imposes the choice of 3 different implementation approaches to obtain features at twice the size.

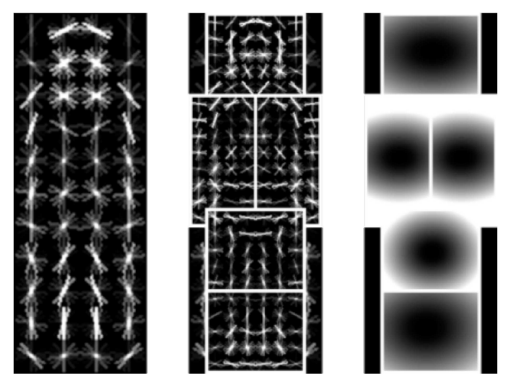

Figure 2: The model used by (Felzenszwalb et al., 2008; Felzenszwalb et al., 2010a). The root-model at the left, the part-models in the middle and the allowed deviation at the right.

The most intuitive manner is to upscale the image where the root model is searched on (coined DPM $U p$ ). Altough the information contained in an image can not be extended, the stability of the model can benefit from this. Another option is to do the opposite, and downscale the image used for the part filters instead (coined DPM Down). Since downscaling is faster than upscaling (both in memory as in computational complexity), this seems a good option. Although it comes with a pitfall. The object can only be found at twice the original size of the model. To obtain the same scale range as the previous design, we have to upscale the image to twice the resolution up front, which takes away the advantage. The last option is to not rescale at all, but use half the amount of pixels to calculate the histogram (coined DPM Half). These three implementation methods are discussed in subsection 3.5, and as can be seen, only the use of taking half the cell-size comes with a minimal accuracy loss, while being a lot less computationally intensive.

We optimised our implementation by eliminating redundant work, exploit locality in memory and avoid global memory for thread-safe code. This allows us to evaluate the layers of the scale-space-pyramid in parallel.

\subsection{Speed, Memory and Accuracy}

In the previous subsections, we described three algorithms seperately. The choice of which algorithm to use independently, or as part of a combination, is based on memory-use, evaluation time and the accuracy. To evaluate these criteria, we use the evaluation framework of (Dollár et al., 2012b; Dollár, 2013) at a Reasonable setting (50px and higher, with max $45 \%$ occlusion). The $120 \mathrm{px}$ height of the Deformable Part Model-model imposes the requirement to perform an initial upscale of 2.4 times (we round to 2.5 times) to obtain detections at 50px. Therefor, we also evaluate the speed and memory-use at different image sizes. All experiments are performed on the same platform. The amount of parallellisation influences both the evaluation speed and memory-use, but the accuracy remains constant.

The HOG-implementation of OpenCV (which we use) has the model embedded in the code, in contrast to our algorithms that uses a text-file. The accuracy we obtain is visualised in figure 3 . As we can observe, the OpenCV-implementation improves its accuracy at higher threshold compared to the original accuracy results.

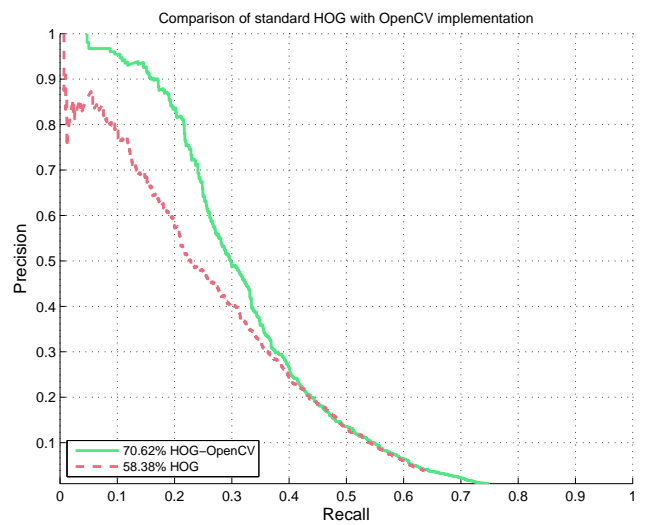

Figure 3: Accuracy comparison between the original HOG and the implementation of OpenCV we use.

We trained an Integral Channel Features model ourself, based on the INRIA pedestrian training set. The complete images are used instead of the normalised ones, and each annotation is rescaled to $41 \times 100$ pixels (size of our inner-model). The outermodel (annotation + extra spacing) is chosen to be $64 \times 128$ pixels, as has been done in the ACF-training code of (Dollár et al., 2014; Dollár, 2013). We used a 2048 stage model, where each stage is a level-two decision tree as weak classifier. To obtain a complete PR-curve, we used a permissive threshold for softcascade. In figure 4 we compare the accuracy results we obtain with the ones in the framework, as obtained by (Dollár et al., 2009). We can observe that our implementation performs slightly better compared to the original Matlab implementation.

The model used for our Deformable Part Model detector is also trained on INRIA, and comes with the original Matlab release (Felzenszwalb et al., 2010b). We used a matlab-script to convert the .mat-file to a textfile which can be used by our implementation. 


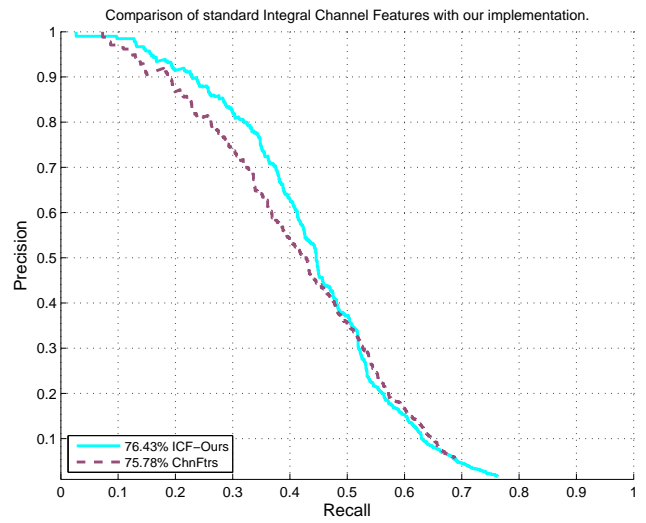

Figure 4: Accuracy comparison between our implementation of Integral Channel Features and the results from (Dollár et al., 2009).

This is a model of $40 \times 120$ pixels. As described in subsection 3.4, there are 3 methods to obtain the features for the evaluation of the root-model and the part-models. In figure 5 we compare these 3 options with the original Matlab-implementation (Latv4-cc) and the implementation of (Dubout and Fleuret, 2012) $(F F L D)$. As we can see, the accuracy results we obtain are all very similar.

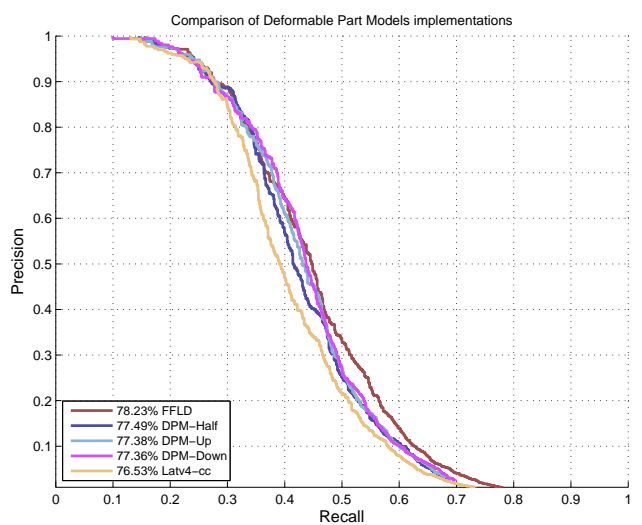

Figure 5: Comparison of the accuracy results of Deformable Part Models.

In table 1 and table 2 we compare the evaluation speed and memory-use respectively, of each implementation of our framework. The improvement in accuracy for both Deformable Part Models and Integral Channel Features over Histogram of Oriented Gradients comes at the cost of speed loss and higher peak memory-use. When we compare our implementations of Deformable Part Models, we can point out that we only need a fraction of the memory needed by FFLD, while using half the cell-size is more than twice as fast on VGA-resolution.

Based on the criteria we just evaluated, we can
Table 1: Comparison of evaluation speed of the algorithms we provide.

\begin{tabular}{c|c|c|c} 
& $640 \times 480$ & $1280 \times 960$ & $1600 \times 1200$ \\
\hline HOG & $15.1 \mathrm{fps}$ & $4.1 \mathrm{fps}$ & $2.66 \mathrm{fps}$ \\
ICF & $10.71 \mathrm{fps}$ & $2.12 \mathrm{fps}$ & $1.37 \mathrm{fps}$ \\
DPM Half & $8.63 \mathrm{fps}$ & $1.30 \mathrm{fps}$ & $0.82 \mathrm{fps}$ \\
DPM Up & $5.58 \mathrm{fps}$ & $0.82 \mathrm{fps}$ & $0.45 \mathrm{fps}$ \\
DPM Down & $2.98 \mathrm{fps}$ & $0.74 \mathrm{fps}$ & $0.48 \mathrm{fps}$ \\
\hline FFLD & $3.828 \mathrm{fps}$ & $0.96 \mathrm{fps}$ & $0.67 \mathrm{fps}$
\end{tabular}

Table 2: Comparison of memory-use when running the pedestrian detection algorithms.

\begin{tabular}{c|c|c|c} 
& $640 \times 480$ & $1280 \times 960$ & $1600 \times 1200$ \\
\hline HOG & $42.9 \mathrm{MB}$ & $141.5 \mathrm{MB}$ & $243.4 \mathrm{MB}$ \\
ICF & $163.1 \mathrm{MB}$ & $446.4 \mathrm{MB}$ & $659.4 \mathrm{MB}$ \\
DPM Half & $82.72 \mathrm{MB}$ & $240.3 \mathrm{MB}$ & $429.2 \mathrm{MB}$ \\
DPM Up & $102 \mathrm{MB}$ & $253 \mathrm{MB}$ & $442 \mathrm{MB}$ \\
DPM Down & $120 \mathrm{MB}$ & $332.6 \mathrm{MB}$ & $537.2 \mathrm{MB}$ \\
\hline FFLD & $790 \mathrm{MB}$ & $3.0 \mathrm{~GB}$ & $4.7 \mathrm{~GB}$
\end{tabular}

select the best pair of detectors to combine. For accuracy, it will be better to combine our Integral Channel Feature-detector with our Deformable Part Modelsdetector, while for speed it may be a better choice to combine Histogram of Oriented Gradients with Integral Channel Features. In section 4 we discuss how to combine detectors, and evaluate the combinations on the same criteria (evaluation speed, peak memoryuse and accuracy) for the pairwise combinations of our implementations.

\section{COMBINATION}

In this section, we will discuss how to combine the detectors we described in section 3. The steps to obtain a combined detection result are visualized in figure 6. The first steps are performed as described in section 3, where a scale-space-pyramid is created from the source image. Each layer of the scale-space pyramid is then processed by a pedestrian detection algorithm. The next step is to normalise the detection scores. This is required, since each detector has a different score-range. The normalisation of detection scores is described in subsection 4.1. After normalisation, we have multiple options. We can just throw the detections (before NMS) in one big pool and treat them as coming from a single detector. This is described in subsection 4.2. A more accurate alternative is performing a smart combination from the detection results after NMS, which is described in subsection 4.3 . 


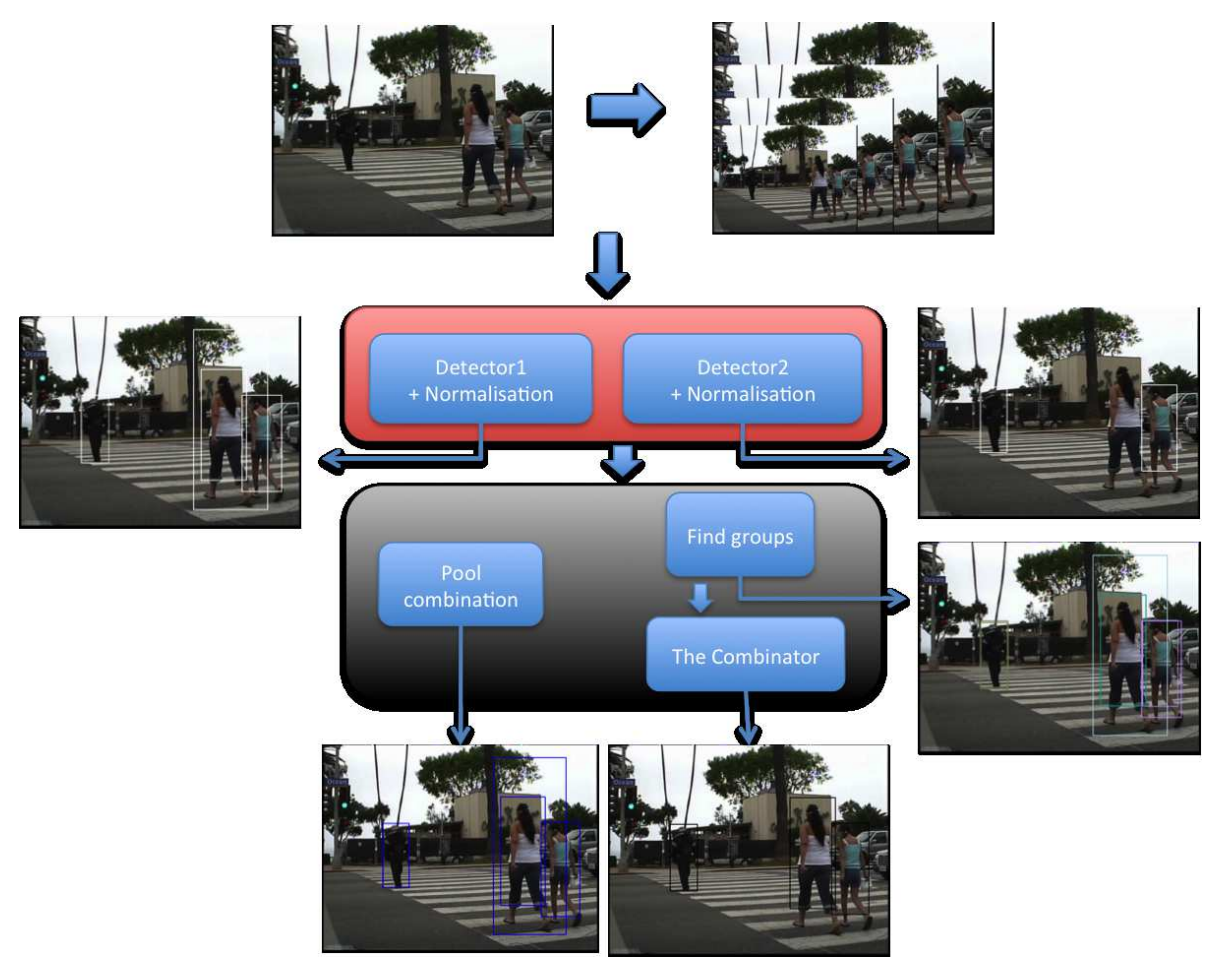

Figure 6: Overview of combination approach.

\subsection{Normalisation}

The normalisation of detection scores is necessary, since the range of detection scores can differ drastically between different detection algorithms. Here we use the standard score approach as has been used by (De Smedt et al., 2014). The equation looks as follows:

$$
S_{\text {norm }}=\frac{\left(S-\mu_{s}\right)}{\sigma_{s}}
$$

This results in all detection scores being positions around the zero-value. For this approach a working point has to be defined and all detections with detection score above the working point are taken into account to calculate the mean and standard deviation.

Next to the score, we also have to equalise the aspect ratio of the bounding boxes between the detectors. The aspect ratio between ICF and DPM (Integral Channel Features and Deformable Part Models) differ slightly. We empirically found out that altering the bounding boxes by keeping the height constant, turns out to aquire the best accuracy results.

\subsection{Pool Combination}

With the normalised scores, we can treat the resulting detections of all detectors equally. This means that we can just put the detections together into the same pool and perform Non-Maximum suppression over all detections found with multiple detectors. As we will show in section 4.4 , the accuracy results depend on the accuracy of the algorithms to combine. Due to normalisation, the detectors are treated equally, so the accuracy difference is lost. The combination approach described in section 4.3 takes this difference into account in the form of the confidence value.

\subsection{The Combinator}

Recently (De Smedt et al., 2014) proposed a technique to combine the results of different object detection algorithm, which they apply for the detection of pedestrian detectors. The combined score they obtained is formed by using a weighted sum of normalised detection scores, where each score is weighted by using two coeficients, the confidence and the complementarity. The confidence is a measurement to express how well a certain detector works on itself, while the complementarity measures the differences between detection results. If detectors are based on completely different feature pools, they most probably will result in different detections, meaning that a combined detection has a higher chance of being correct, compared to very similar detectors leading to the same detection. They use the following equation to 
obtain the final detection score:

$$
S_{\text {final }}=\sum_{i=1}^{n} c_{\operatorname{conf}(i)} c_{\operatorname{compl}(i)}\left(S_{i}+Q\right)
$$

The $Q$ added to the normalised score is required when the Standard score approach is used for normalisation, to avoid the presence of negative normalised scores, which would lead to a decrease in the weighted sum formula.

The same working point is used to aquire the mean and standard deviation for normalisation (as described in subsection 4.1) as to obtain the confidence and complementarity coeficients. The confidence is defined as the area between the origin and the working point. Here, we simplify the weighted sum formula by eliminating the use of a complementarity value, since it has no additional information when used for a pairwise combination.

\subsection{Evaluation of Speed and Accuracy}

Finally, we compare the implementations we have proposed as part of our framework. The accuracy of the object detection algorithms, and the models we use, is already discussed in subsection 3.5, but are shown again in comparison with the combination techniques we described earlier. In figure 7 we compare the use of pool-combination as described in subsection 4.2. As we can observe, the combination of Histogram of Oriented Gradients with either Deformable Part Models or Integral Channel Features does not improve accuracy. This is due to the fact that the accuracy-difference is ignored. When we combine Integral Channel Features and Deformable Part Models on the other hand, which have more or less an equal accuracy, we obtain a big improvement in accuracy.

In figure 8 , we compare the accuracy results we

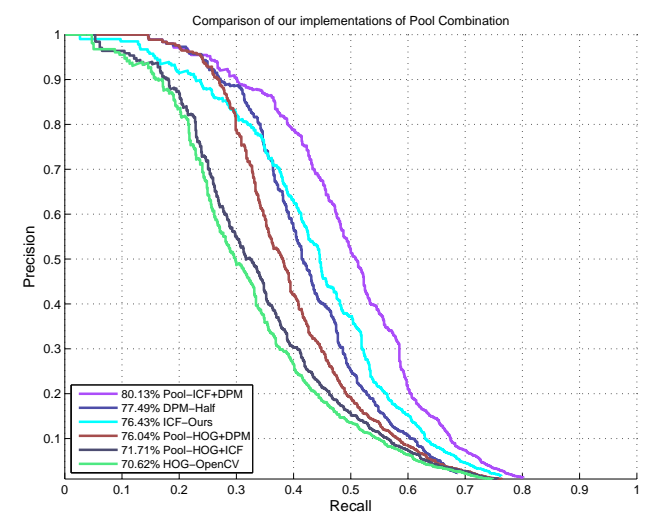

Figure 7: Comparison of the accuracy of the implementations we propose.

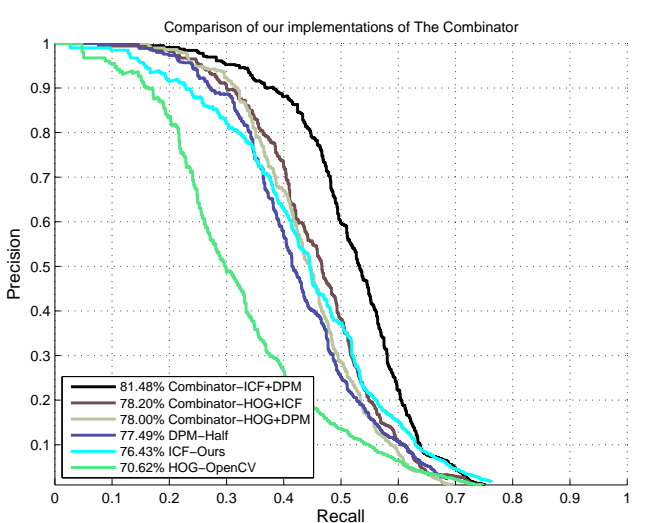

Figure 8: Comparison of the accuracy of the implementations we propose.

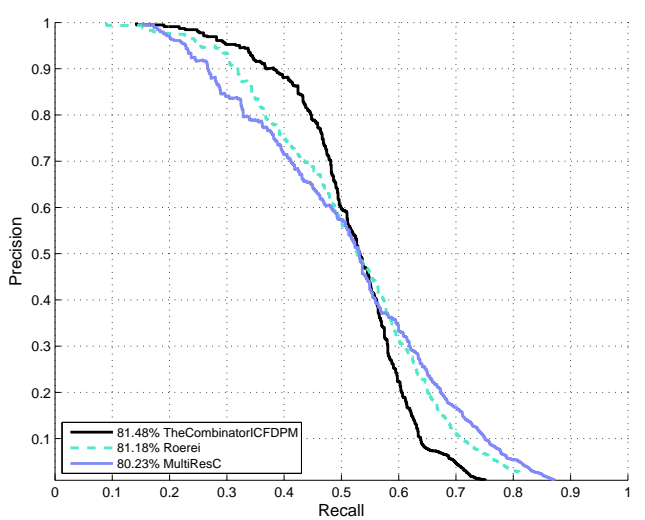

Figure 9: Comparison of the our most accurace combination with the state-of-the-art.

obtain by using the smarter combination approach described in subsection 4.3. Here we can point out that the accuracy benefits from combining. The additional value of Histogram of Oriented Gradients is not that big though.

Table 3 and table 4 show the evaluation speed and peak memory-use respectively of our combination approaches. As we can point out, the evalutation speed and peak-memory of a combination is dependend on the slowest algorithm in the combination. When for example Integral Channel features is combined with Deformable Part Models, part of the CPU-cores are assigned to the Deformable Part Models algorithm. Since Deformable Part Models does not need as much memory, the peak memory-use is less then the sum of Integral Channel Features and Deformable Part Models seperately.

When we compare the combination of Deformable Part Models and Integral Channel Features with the state-of-art detectors (figure 9), it can be seen that a combination reaches impressive accury results. Altough the PR-curve crosses the ones of Roerei (Be- 
nenson et al., 2013) and MultiRes (Park et al., 2010), it is only at the point where only half the detections are correct (precision of 0.5). For most applications the required precision is significantly higher. For MultiRes, no evaluation speed is mentioned in (Park et al., 2010), and (Benenson et al., 2013) (the Roerei-detector) claims an evaluation speed of $5 \mathrm{~Hz}-$ $20 \mathrm{~Hz}$ while using GPU-hardware.

Table 3: Comparison of evaluation speed of the combined algorithms.

\begin{tabular}{c|c|c|c} 
& $640 \times 480$ & $1280 \times 960$ & $1600 \times 1200$ \\
\hline HOG & $15.1 \mathrm{fps}$ & $4.1 \mathrm{fps}$ & $2.66 \mathrm{fps}$ \\
ICF & $10.71 \mathrm{fps}$ & $2.12 \mathrm{fps}$ & $1.37 \mathrm{fps}$ \\
DPM Half & $8.63 \mathrm{fps}$ & $1.30 \mathrm{fps}$ & $0.82 \mathrm{fps}$ \\
\hline Pool $H O G+D P M$ & $6.8 \mathrm{fps}$ & $0.57 \mathrm{fps}$ & $0.31 \mathrm{fps}$ \\
Pool $H O G+I C F$ & $7.65 \mathrm{fps}$ & $1.60 \mathrm{fps}$ & $1.03 \mathrm{fps}$ \\
Pool $I C F+D P M$ & $7 \mathrm{fps}$ & $0.54 \mathrm{fps}$ & $0.29 \mathrm{fps}$ \\
\hline Combinator $H O G+D P M$ & $6.8 \mathrm{fps}$ & $0.57 \mathrm{fps}$ & $0.31 \mathrm{fps}$ \\
Combinator $H O G+I C F$ & $7.57 \mathrm{fps}$ & $1.57 \mathrm{fps}$ & $1.03 \mathrm{fps}$ \\
Combinator $I C F+D P M$ & $6.78 \mathrm{fps}$ & $0.53 \mathrm{fps}$ & $0.29 \mathrm{fps}$
\end{tabular}

Table 4: Comparison of memory-use when running the pedestrian detection algorithms.

\begin{tabular}{c|c|c|c} 
& $640 \times 480$ & $1280 \times 960$ & $1600 \times 1200$ \\
\hline HOG & $42.9 \mathrm{MB}$ & $141.5 \mathrm{MB}$ & $243.4 \mathrm{MB}$ \\
ICF & $163.1 \mathrm{MB}$ & $446.4 \mathrm{MB}$ & $659.4 \mathrm{MB}$ \\
DPM Half & $82.72 \mathrm{MB}$ & $240.3 \mathrm{MB}$ & $429.2 \mathrm{MB}$ \\
\hline Pool $H O G+D P M$ & $80.2 \mathrm{MB}$ & $261 \mathrm{MB}$ & $424 \mathrm{MB}$ \\
Pool $H O G+I C F$ & $162 \mathrm{MB}$ & $428 \mathrm{MB}$ & $657 \mathrm{MB}$ \\
Pool $I C F+D P M$ & $105 \mathrm{MB}$ & $318 \mathrm{MB}$ & $505 \mathrm{MB}$ \\
\hline Combinator $H O G+D P M$ & $82.2 \mathrm{MB}$ & $264 \mathrm{MB}$ & $424 \mathrm{MB}$ \\
Combinator $H O G+I C F$ & $174 \mathrm{MB}$ & $420 \mathrm{MB}$ & $694 \mathrm{MB}$ \\
Combinator $I C F+D P M$ & $124 \mathrm{MB}$ & $336 \mathrm{MB}$ & $482 \mathrm{MB}$
\end{tabular}

\section{CONCLUSION}

In this paper we present for the first time a fullpipeline implementation of detection combination as an open framework. In contrast to the traditional approach of improving detection accuracy by optimising a single detector, we use a technique of combining multiple pedestrian detectors instead, a technique proposed in (De Smedt et al., 2014). Herefor we use the Histogram of Oriented Gradients implementation of OpenCV with our own implementation of Integral Channel Features and of Deformable Part Models. Based on the criteria of evaluation speed, peak memory-use and accuracy, we obtained superior results to publicly available (CPU) implementations. The accuracy obtained by combining Deformable Part Models with Integral Channel Features is impressive compared to state-of-the-art detectors which are far more computation intensive.

Code for this framework is available at http:// eavise.be/AbnormalBehaviour, and can be used for research purposes.

\section{REFERENCES}

Benenson, R., Mathias, M., Timofte, R., and Van Gool, L. (2012). Pedestrian detection at 100 frames per second. In $C V P R$. IEEE.

Benenson, R., Mathias, M., Tuytelaars, T., and Van Gool, L. (2013). Seeking the strongest rigid detector. In CVPR. IEEE.

Benenson, R., Omran, M., Hosang, J., and Schiele, B. (2014). Ten years of pedestrian detection, what have we learned?

Bourdev, L. and Brandt, J. (2005). Robust object detection via soft cascade. In $C V P R$, volume 2. IEEE.

Dalal, N. and Triggs, B. (2005). Histograms of oriented gradients for human detection. In $C V P R$, volume 1 . IEEE.

De Smedt, F., Struyf, L., Beckers, S., Vennekens, J., De Samblanx, G., and Goedemé, T. (2012). Is the game worth the candle? evaluation of opencl for object detection algorithm optimization. PECCS.

De Smedt, F., Van Beeck, K., Tuytelaars, T., and Goedemé, T. (2013). Pedestrian detection at warp speed: Exceeding 500 detections per second. In CVPRW. IEEE.

De Smedt, F., Van Beeck, K., Tuytelaars, T., and Goedemé, T. (2014). The combinator: optimal combination of multiple pedestrian detectors. In ICPR.

Dollár, P. (2013). Piotrs image and video matlab toolbox (pmt). Software available at: http://vision. ucsd. edu/ pdollar/toolbox/doc/index. html.

Dollár, P., Appel, R., Belongie, S., and Perona, P. (2014). Fast feature pyramids for object detection. PAMI, 36(8).

Dollár, P., Appel, R., and Kienzle, W. (2012a). Crosstalk cascades for frame-rate pedestrian detection. In ECCV.

Dollár, P., Belongie, S., and Perona, P. (2010). The fastest pedestrian detector in the west. In $B M V C$.

Dollár, P., Tu, Z., Perona, P., and Belongie, S. (2009). Integral channel features. In $B M V C$, volume 2.

Dollár, P., Wojek, C., Schiele, B., and Perona, P. (2012b). Pedestrian detection: An evaluation of the state of the art. PAMI, 34.

Dubout, C. and Fleuret, F. (2012). Exact acceleration of linear object detectors. In ECCV. Springer.

Felzenszwalb, P., McAllester, D., and Ramanan, D. (2008). A discriminatively trained, multiscale, deformable part model. In CVPR. IEEE.

Felzenszwalb, P. F., Girshick, R. B., and McAllester, D. (2010a). Cascade object detection with deformable part models. In CVPR. IEEE.

Felzenszwalb, P. F., Girshick, R. B., and McAllester, D. (2010b). Discriminatively trained deformable part models, release 4. http://people.cs.uchicago.edu/ pff/latent-release4/.

Mathias, M., Benenson, R., Timofte, R., and Gool, L. V. (2013). Handling occlusions with franken-classifiers. In ICCV. IEEE.

Park, D., Ramanan, D., and Fowlkes, C. (2010). Multiresolution models for object detection. In ECCV. Springer. 